\title{
Diammonium Glycyrrhizinate
}

National Cancer Institute

\section{Source}

National Cancer Institute. Diammonium Glycyrrhizinate. NCI Thesaurus. Code C102865.

The diammonium salt of glycyrrhizin and the active constituent in the traditional Chinese medicinal herb Glycyrrhiza uralensis (Chinese liquorice or Gan-Cao) with antiinflammatory, antioxidant and hepatoprotective properties. Diammonium glycyrrhizinate (DG) is slowly metabolized within the cells into glycyrrhetic acid, which inhibits enzymes that control cortisol metabolism and contributes to this agent's anti-inflammatory effect. Although the exact mechanism of action remains to be fully elucidated, DG may prevent or reduce hepatotoxicity via the scavenging of free radicals. This agent also upregulates the expression of transcription coactivator PGC-1alpha and modulates hepatic enzymes such as alanine aminotransferase (ALT), aspartate aminotransferase (AST), superoxide dismutase and glutathion peroxidase. 